\title{
LC Headings from December 2018 and January and February 2019 Lists
}

Compiled by Ann Heinrichs

The new headings listed here reflect the most recent information available at the time of publication. Items in this list were selected from 2018 list number 12 (December 14) and 2019 list numbers 01 (January 18) and 02 (February 15).

\section{SUBJECT HEADINGS}

150 Alevi women [May Subd Geog] [sp2018002884]

053 HQ1170.2

550 BT Muslim women

150 Alevis--Persecutions [May Subd Geog] [sp2018003199]

550 BT Persecution

150 Alevis--Persecutions--Turkey [sp2018003198]

150 Brown Memorial Tower (Union Theological Seminary, New York, N.Y.) [sp2018003177]

450 UF Brown Tower (Union Theological Seminary, New York, N.Y.)

550 BT Towers--New York (State)

510 BT Union Theological Seminary (New York, N.Y.)

150 Buddhist sermons, Mon [May Subd Geog] [sp2018002218]

450 UF Mon Buddhist sermons

150 Catechisms, Tahitian [sp2018002538]

450 UF Tahitian catechisms

150 Catholic lesbians [May Subd Geog] [sp2018002746]

$450 \quad$ UF Lesbian Catholics

550 BT Lesbians

150 Catholic sexual minorities [May Subd Geog] [sp2018002722]

450 UF Sexual minority Catholics

550 BT Sexual minorities

150 Christian lesbians [May Subd Geog] [sp2006008772]

681 Example under Sexual minorities ADD FIELD 
150 Cooking (Matzos) [May Subd Geog] [sp2018003120]

450 UF Cooking with matzos

450 UF Matzos--Use in cooking

$550 \quad$ BT Cooking (Bread)

150 Covens [May Subd Geog] [sp 96010537]

550 BT Meetings DELETE FIELD

550 BT Religious gatherings ADD FIELD

150 Czech American Catholics [May Subd Geog] [sp2018003054]

053 BX1407.C9

450 UF Catholics, Czech American

550 BT Catholics--United States

150 Data services librarians [May Subd Geog] [sp2018002071]

$053 \quad$ Z682.4.D38

$450 \quad$ UF Data librarians

550 BT Librarians

680 Here are entered works on librarians engaged in managing data, using data as a resource and/ or supporting patrons working with research data or data sets.

150 Donkeys in the Bible [sp2018002828]

150 Embedded librarians [May Subd Geog] [sp2018002244]

053 Z682.4.E46

$550 \quad$ BT Librarians

680 Here are entered works on librarians who are integrated with a user group to offer direct research assistance and often work in the same physical location as the group they serve.

150 Esbat (Neopagan custom) [May Subd Geog] [sp2018002582]

550 BT Neopaganism--Customs and practices

680 Here are entered works on covens' ritual observance of the full moon, and sometimes also the dark moon and/or first and last quarters. Works on the neopagan religious festivals that commemorate the phases of the changing seasons are entered under Sabbat.

681 Note under Sabbat

150 Georgian (Wiccan sect) [May Subd Geog] [sp2018002539]

$550 \quad$ BT Wiccan sects

150 Hindu hymns, Malayalam [May Subd Geog] [sp2018002401]

450 UF Malayalam Hindu hymns

150 Hymns, Ganda [sp2018002793]

450 UF Ganda hymns

150 Imaginary companions--Religious aspects [sp2018002435] 
150 Ismailites [May Subd Geog] [sp 85068625]

450 UF Ismaili DELETE FIELD

450 UF Ismailis ADD FIELD

150 James Tower (Union Theological Seminary, New York, N.Y.) [sp2018003178]

450 UF James Chapel Tower (Union Theological Seminary, New York, N.Y.)

550 BT Towers--New York (State)

510 BT Union Theological Seminary (New York, N.Y.)

150 Jewish museums [May Subd Geog] [sp 85070308]

450 UF Judaica museums ADD FIELD

450 UF Judaism--Museums ADD FIELD

150 Ladino language [May Subd Geog] [sp 85073893 ]

450 UF Judesmo DELETE FIELD

450 UF Judezmo DELETE FIELD

450 UF Haketia language (Ladino) ADD FIELD

450 UF Hakitia language (Ladino) ADD FIELD

450 UF Judeo-Castilian language ADD FIELD

450 UF Judeo-Espagnol language ADD FIELD

450 UF Judeo-Espanyol language ADD FIELD

450 UF Judesmo language ADD FIELD

450 UF Judezmo language ADD FIELD

450 UF Ladino Sephardic language ADD FIELD

450 UF Sefardi language ADD FIELD

450 UF Sephardi language ADD FIELD

450 UF Sephardic language ADD FIELD

551 BT Israel--Languages ADD FIELD

551 BT Turkey--Languages ADD FIELD

150 Lammas [May Subd Geog] [sp 00009786]

550 BT Calendar, Celtic ADD FIELD

150 Madrasah buildings--Turkey [sp2018003174]

150 Madrasahs [May Subd Geog] [sp 96009872 ]

450 UF Madrasehs ADD FIELD

450 UF Medressehs ADD FIELD

150 Mercy in art [Not Subd Geog] [sp2018002875]

150 Muslim lesbians [May Subd Geog] [sp2018001970]

550 BT Lesbians

150 Neopaganism--Customs and practices [sp2018003113] 
150 Prostitutes in rabbinical literature [sp2018002734]

053 BM496.9.P76

550 BT Rabbinical literature

151 Ruta del Peregrino (Mexico) [sp2018002967]

451 UF Peregrino, Ruta del (Mexico)

451 UF Pilgrim Route (Mexico)

451 UF Pilgrim's Route (Mexico)

451 UF Ruta Peregrino (Mexico)

550 BT Trails--Mexico

781 Mexico--Ruta del Peregrino

150 Sabbat [May Subd Geog] [sp 90004592 ]

550 BT Fasts and feasts--Neopaganism ADD FIELD

680 Here are entered works on the neopagan religious festivals that commemorate the phases of the changing seasons. Works on covens' ritual observance of the full moon, and sometimes also the dark moon and/or first and last quarters, are entered under Esbat (Neopagan custom). ADD FIELD

681 Note under Esbat (Neopagan custom) ADD FIELD

150 Saint Brigid's Day [May Subd Geog] [sp 96002699]

053 GT4995.B74 DELETE FIELD

053 BF1572.S325 (Witchcraft) ADD FIELD

053 GT4995.B74 (Manners and customs) ADD FIELD

$450 \quad$ UF Saint Brighid's Day ADD FIELD

550 BT Festivals--Ireland DELETE FIELD

550 BT Calendar, Celtic ADD FIELD

550 BT Fasts and feasts ADD FIELD

550 BT Spring festivals ADD FIELD

150 Samhain [May Subd Geog] [sp 96002291]

053 BF1572.S35 DELETE FIELD

053 BF1572.S35 (Witchcraft) ADD FIELD

550 BT Fasts and feasts--Neopaganism DELETE FIELD

550 BT Fasts and feasts ADD FIELD

150 Shiite women [May Subd Geog] [sp2018002952]

550 BT Muslim women

150 Signs and symbols--Religious aspects [sp2018000930]

150 Signs and symbols--Religious aspects--Protestant churches [sp2018000931] 
150 Solitary practitioners (Wiccans) [May Subd Geog] [sp2018002583]

450 UF Hedge witches

450 UF Solitary witches

550 BT Wiccans

550 BT Witches

150 Transgender librarians [May Subd Geog] [sp2007003740]

681 Example under Sexual minorities ADD FIELD

150 Transnationalism--Religious aspects [sp2018002916]

150 Transnationalism--Religious aspects--Catholic Church [sp2018002917]

150 Transnationalism--Religious aspects--Hinduism [sp2018002920]

150 Virtual reality--Library applications ～[May Subd Geog] [sp2018002574]

$053 \quad$ Z678.93.S53

150 Witches in rabbinical literature [sp2018002732]

$053 \quad$ BM496.9.W58

550 BT Rabbinical literature

150 Yule (Festival) [May Subd Geog] [sp2015001181]

053 BF1572.Y85 DELETE FIELD

053 BF1572.Y85 (Witchcraft) ADD FIELD

550 BT Calendar, Celtic ADD FIELD

\section{GENRE/FORM TERMS}

155 Fables [gp2014026327]

455 UF Moral and philosophical stories DELETE FIELD

455 UF Fabular tales ADD FIELD

455 UF Moral and philosophical tales ADD FIELD

680 Stories intended to teach moral lessons, and whose main characters are generally animals or inanimate objects that speak and act like human beings. DELETE FIELD

680 Stories intended to teach moral lessons, and whose main characters are generally animals or inanimate objects that speak and act like human beings. For short, simple stories that convey a moral lesson, and whose main characters are generally humans, see Parables. ADD FIELD

681 Note under Parables ADD FIELD 
155 'Īd al-Aḍhā fiction [gp2018026139]

455 UF Eid al-Adha fiction

455 UF Eid fiction

455 UF Eid ul-Adha fiction

455 UF ' '̄d al-Kabīr fiction

455 UF 'I $\mathrm{I} d$ al-Nahr fiction

455 UF 'I d al-Qurbān fiction

555 BT Religious fiction

155 'İd al-Fiṭr fiction [gp2018026138]

455 UF Eid al-Fitr fiction

455 UF Eid fiction

455 UF Eid ul-Fitr fiction

455 UF 'Īd al-Ṣadaqah fiction

455 UF 'Īd al-Ṣaghìr fiction

455 UF 'Īd-ul-Fitr fiction

555 BT Religious fiction

155 Parables [gp2014026467]

455 UF Parabolic stories ADD FIELD

680 Short, simple stories that convey a moral lesson. DELETE FIELD

680 Short, simple stories that convey a moral lesson, and whose main characters are generally humans. For stories intended to teach moral lessons, and whose main characters are generally animals or inanimate objects that speak and act like human beings, see Fables. ADD FIELD

681 Note under Fables ADD FIELD

155 Radio sermons CANCEL HEADING [gp2011026512]

682 This authority record has been deleted because the term is covered by the genre/form terms Sermons (DLC)gf201502051 and Religious radio programs (DLC)gf2011026527

155 Religious materials [gp2015026026]

680 Collections of religious materials that are composed of multiple genres and/or forms to which more specific headings such as Apocryphal works cannot be applied. DELETE FIELD

155 Religious radio programs [gp2011026527]

455 UF Radio sermons [Former heading] ADD FIELD

155 Sermons [gp2015026051]

455 UF Radio sermons [Former heading] ADD FIELD 